\title{
Cost-effectiveness of Ibrutinib as Frontline Treatment For Adult Patients With Chronic Lymphocytic Leukaemia in Belgium
}

\author{
Ann Smet ${ }^{1}$, Siyang Peng ${ }^{2}$, Emily Dorman², Kristen Deger ${ }^{2}$, Sonja Sorensen², Simona Baculea ${ }^{3}$, Sarah Cote ${ }^{3}$ \\ ${ }^{1}$ Janssen-Cilag NV, Beerse, Belgium; ${ }^{2}$ Evidera, Bethesda, MD, USA; ${ }^{3}$ Janssen-Cilag, High Wycombe, Bucks, UK
}

\section{BACKGROUND AND OBJECTIVE}

- Chronic lymphocytic leukemia (CLL) is an incurable and life-threatening orphan disease that most commonly affects the elderly. Choice of treatment, especially for those with comorbidities, requires a trade-off between efficacy and toxicity. This study assessed the costeffectiveness - from a Belgian healthcare payer perspective_of ibrutinib, a first-in-class covalent Bruton's tyrosine kinase inhibitor associated with significant improvement in progression-free survival (PFS) and overall survival (OS) in frontline treatment of elderly/unfit patients with CLL in terms of projected life-years (LY), quality-adjusted LYS (QALY), and incremental cost-effectiveness ratios (ICER).

\section{METHODS}

- A survival partition model with three health states (PFS, post-progression survival [PPS], and death) was developed to evaluate the health and cost consequences of CLL treatment (Figure 1) with ibrutinib vs. chlorambucil monotherapy, chlorambucil + obinutuzumab (Ch+O), bendamustine + rituximab (BR), and physician's choice (PC), a mixed basket of the above treatments, as used in Belgian clinical practice.

- A cohort of elderly, unfit, treatment-naïve patients were followed for 25 years (reflective of a lifetime time horizon), and health and cost outcomes were discounted by $1,5 \%$ and $3,0 \%$, respectively. ${ }^{1}$ Patients entered the model and initiated frontline treatment for CLL. Upon progression, patients transitioned to PPS, where they were modelled to receive a subsequent line of active treatment that impacted cost but not patient survival. Patients could die at any time in the model.

\section{Data Sources}

- Efficacy of ibrutinib and chlorambucil was informed by RESONATE-2 trial data. ${ }^{2}$ Efficacy of comparators were based on a Bayesian network meta-analysis (NMA) that estimated the treatments' relative efficacy (PFS and OS hazard ratios [HR]) based on data from a systematic literature review (SLR) (Table 1). ${ }^{3}$ Baseline utility and changes in utility associated with ibrutinib treatment were based on EuroQoL-Five Dimensions data collected in RESONATE-2 (Table 2). Other utility values were based on published sources identified through the SLR.

- Medical resource use was informed by clinical expert opinion, and unit costs were sourced from Belgian cost databases.

\section{Projection of OS and PFS}

- PFS and OS outcomes were projected from clinical trials using standard parametric fittings to establish reference curves that represent the underlying risk of progression and/or death. PFS and OS HRs derived from the NMA were applied to the reference curve to estimate the relative efficacy in terms of PFS and OS for each treatment arm in the model. ${ }^{3}$

PFS reference curve: CLL11 chlorambucil PFS curve, Weibull projection (best fit per Akaike and Bayesian Information Criterion statistics)

OS reference curve: RESONATE-2 ibrutinib OS curve, capped by the Belgian general population mortality.

\section{Figure 1. Model Diagram}

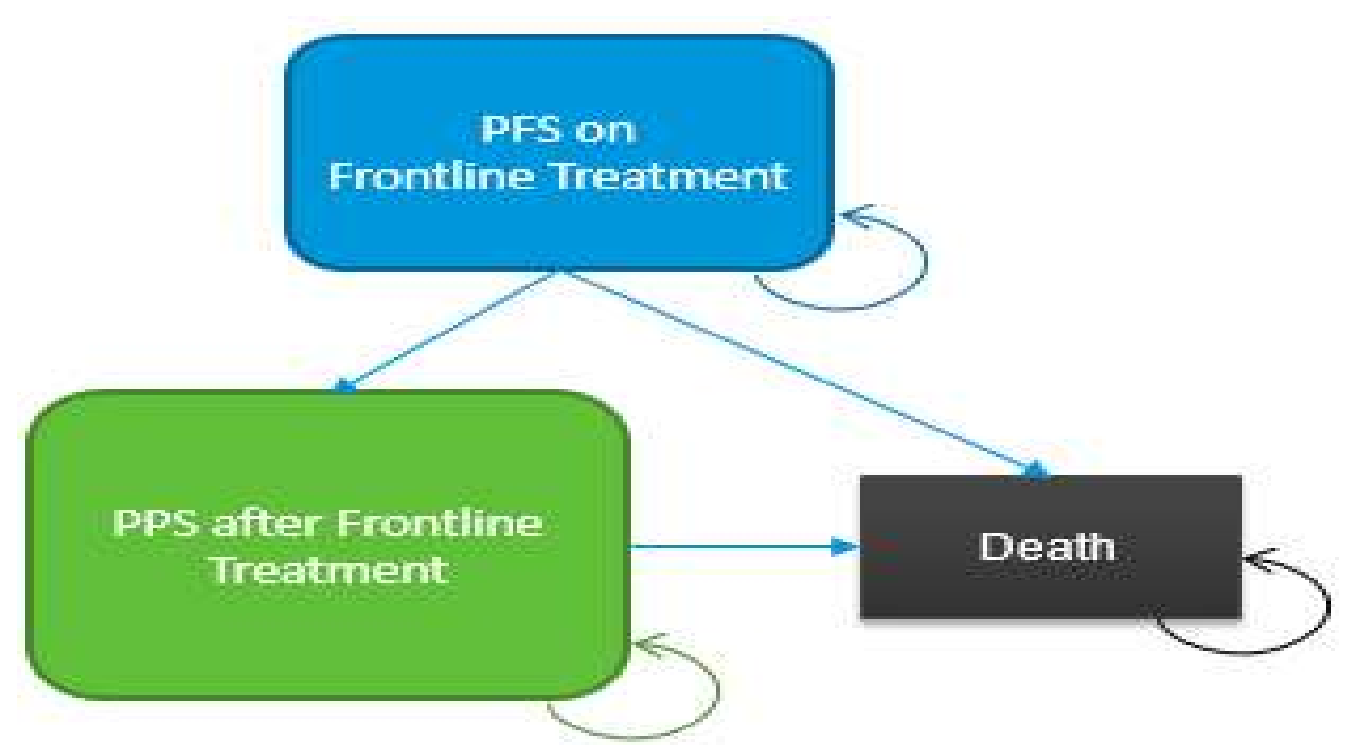

Table 1. Comparative Efficacy based on NMA

\begin{tabular}{|c|c|c|c|}
\hline vs. Chlorambucil & OS HR [95\% Cl] & PFS HR, [95\% Cl] & Reference \\
\hline Ibrutinib & $0,16[0,05,0,55]$ & $0,16[0,09,0,28]$ & \multirow{3}{*}{ Xu Y et al. $2017^{3}$} \\
\hline $\mathrm{Ch}+\mathrm{O}$ & $0,41[0,23,0,74]$ & $0,19[0,14,0,27]$ & \\
\hline BR & $0,64[0,28,1,50]$ & $0,24[0,14,0,40]$ & \\
\hline
\end{tabular}

Table 2. Utility Values Used in Model

\begin{tabular}{|r|c|c|}
\hline Baseline utility, frontline PFS & 0,780 & RESONATE-2 EQ-5D \\
\hline Utility change due to ibrutinib treatment & 0,036 & RESONATE-2 EQ-5D \\
\hline Utility change due to IV administration & $-0,040$ & Obinutuzumab NICE ERG \\
\hline Utility change due to hospital visits* & $-0,120$ & Obinutuzumab NICE ERG \\
\hline *applied first administration of Ch+O only & \multicolumn{2}{|l}{} \\
\hline
\end{tabular}

RESULTS

- Deterministic results indicated that ibrutinib was associated with health gains of up to 7,13 LYs and 4,74 QALYs, and higher costs, which were largely driven by ibrutinib's superior PFS (i.e., longer time on treatment) (Table 3). Deterministic sensitivity analyses indicated that model results were sensitive to key drivers, including discount rates for health and cost outcomes, and ibrutinib treatment duration.

Table 3. Results: Incremental Outcomes vs. Ibrutinib

\begin{tabular}{|l|c|c|c|c|}
\hline Ibrutinib vs. Comparator & \multicolumn{1}{c}{ Chlorambucil } & \multicolumn{1}{c|}{ BR } & \multicolumn{1}{c|}{ Ch+O } & PC \\
\hline Incremental cost $(€)$ & 244.862 & 226.063 & 203.062 & 217.174 \\
\hline Incremental LYs & 7,13 & 5,72 & 4,11 & 5,11 \\
\hline Incremental QALYs & 4,74 & 3,68 & 2,66 & 3,30 \\
\hline ICER per LY gained $(€)$ & 34.361 & 39.523 & 49.424 & 42.540 \\
\hline ICER per QALY gained $(€)$ & 51.641 & 61.506 & 76.451 & 65.760 \\
\hline
\end{tabular}

\section{CONCLUSIONS}

- Results indicate that ibrutinib offers a pronounced benefit over comparators for frontline treatment of CLL.

ICERs are well within 1-3-times Belgium's gross domestic product (€42.120 - €126.359), ${ }^{6,7}$ a standard metric of value for money, ${ }^{8}$ demonstrating that ibrutinib is a cost-effective resource for elderly/unfit frontline-treated CLL patients.

\section{REFERENCES}

1. Cleemput I, et al. KCE Reports 78C (D/2008/10.273/27) 2008; https://kce.fgov.be/sites/default/files/page_documents/d20081027327.pdf

2. Burger JA, et al. N. Engl. J. Med. 2015;373(25):2425-2437.

3. Xu Y, et al. J Comp Eff Res. 2017.

4. Pharmacyclics LLC. CSR PCYC-1115-CA. May 2015.

5. NICE. Appraisal consultation document: Obinutuzumab in combination with chlorambucil for untreated chronic lymphocytic leukaemia. 2014.

6. OECD. Gross Domestic Product. 2016. https://data.oecd.org/gdp/gross-domestic-product-gdp.htm

7. OANDA. Average 2016 USD to Euro conversion factor: 0.90372. 2016. https://www.oanda.com/currency/average

8. Commission on Macroeconomics. Macroeconomics and Health: Investing in Health for Economic Development. Prepared for WHO. 2001. 